\title{
Tasks of some dietary essential nutrients on epigenetics and one carbon metabolism in livestock during production
}

\author{
Mohamed S. Yusuf ${ }^{1,2 a}$, Ahmed Kamel El-Sayed ${ }^{2 b}$, Amr, A. Hashish ${ }^{3}$, Zhen Sun ${ }^{1}$, Hengmi \\ $\mathrm{Cui}^{1^{*}}$ \\ ${ }^{1}$ Yangzhou University, Institute of Epigenetics and Epigenomics, Yangzhou 225009, Jiangsu, China. \\ ${ }^{2}$ Suez Canal University, 41522 Faculty of Veterinary Medicine, Ismailia, Egypt. a Department of Nutrition and Clinical \\ Nutrition. b Department of Anatomy and Embryology. 3Reference Laboratory for Veterinary quality Control of Poultry \\ Production. \\ *Corresponding author, $\underline{\text { hmcui@yzu.edu.cn }}$
}

\begin{abstract}
Interchanges in the epigenome caused by the environment have been cited in multiple animal being, ranging from insects to rodents passing through poultry, larger animals, and nonhuman primates to humans. These include DNA methylation, chromatin remodeling, histone modifications, and more recently the index has magnified to incorporate noncoding RNAs and microRNA gene regulation. Thus, it is increasingly accepted that, nutrition one of the factors that alter gene expression and affect's health during animal productivity through epigenetic modification. DNA methylation is the most widely studied form of epigenetic modification and occurs within the one-carbon metabolism tract which is dependent upon a number of enzymes in the presence of micronutrients, including folate, choline, and betaine acquired through the diet. In vertebrates DNA methylation is primarily a stable repressive mark built at cytosines in CpG dinucleotides; however, its regulation is more powerful than previously believed. Nutri-Epigenomic is an emerging discipline examining the role of nutrient on gene expression. Ultimately, DNA methylation and other epigenetic status, as well as dietetic practices, particularly micronutrient intake, may influence health and productive phenotypes, also playing as significant biomarker in additive-benefit assessment during the period of use. There are a tight interrelationship betaine, choline and methionine. All are dietary sources of methyl groups made it prominent when studying diet-DNA methylation. In this review, our team will focus on the role of important methyl donors and nutrient-gene inter actions through the one-carbon metabolism.
\end{abstract}

KeywordsNutri-Epigenomics, Livestock, methyl donors, one carbon metabolism.

\section{Introduction}

Since 1940s the term epigenetics was first proposed by Waddington, where, it was early used to describe a new phenotype due to gene-environment interaction. That way indicative the source of the phenotype from the genotype, a procedure that is also affected by the environment. As from 20 years ago, epigenetic trends had become in increasing way every day, sharing new sciences serving human health, pathogenesis of diseases, embryogenesis, agriculture sciences, including plant and animal productivity as well as animal models used to enhance the human researches. Many definition regarding epigenetic, were discussed in many articles with the same strategy of changes in gene function (expression), with new phenotype due to changes in gene physiology other than anatomical (sequence) feature. Epigenetic, is investigating and analyzing mitotically and/or meiotically inherited changes in gene function,that areaway from changes in gene sequence[1,2]. Also, "Any long-term change in gene function that does not involve a change in gene sequence or structure" [3]. There are a continuous conversation among cellular elements and the surrounding environment, epigenetic modifications are dynamic throughout the lifecourse and can be heavily influenced by external factors, and thus, external effects on the epigenome may alter gene expression, potentiallygiving rise to new phenotype. In a brief, DNA and chromatin alterations that remain from a cell division to the subsequent one, regardless of changes in the basic DNA sequence, known as epigenetics. Whereas, the in general epigenetic status of a cell that suits as an interface between the genome and the surround environment referred as epigenome. The dynamic character of epigenetic rule, unlike the static nature of the gene sequence allows a mechanism for reprogramming gene function in response to environmental changes, including diet. Thus, epigenetics might give an interpretation for well recorded gene $\mathrm{x}$ environment interactions[3]. Diet and its fine elements are the most environmental factors controlling intensity of gene expression, for example, honeybees grow to be either queens or workers relying on whether they are fed royal jelly or beebread. Despitebeing genetically equal at the larvae level, honeybees fed pure royal jelly are obviously different from worker honeybees. The different honeybeephenotype occurs as a result of epigenetic modifications in DNA methylation styles induced by the kind of honey they are fed with, therefore, showing a strong link between diet-induced epigenetic modifications that lead to unusual development from the same genetic background. In addition, other reports in mammals revealed that environmental stimuli -different dietary factors- can modify DNA methylation and histone acetylation models and, accordingly, influence gene regulation and the phenotypical expression ofa gene[4].Some of the dietary micro-elements were revealed documented phenotypes due to their epigenetic effect, so in this review we would show basic epigenetic machineries beside the essential role of some methyl donators that affecting gene expression away from changes in gene sequence.

\section{Epigenetic machineries}

DNA as well as non-coding RNA methylation and histone post-translational modifications are important epigenetic mechanisms regulating gene activity. 


\subsection{DNA Methylation}

In mammals, methylation of DNA is an epigenetic adaptation that commonly exists at 5-methylcytosine within $\mathrm{CpG}(\mathrm{C}$ followed by $\mathrm{G}$ ) dinucleotide (Regions of the genome rich in $\mathrm{CpG}$ dinucleotide often located at the promoters of genes) and it is an essential tool for epigenetic regulation of the genes expression. Methylation of $\mathrm{CpG}$ within these regions is generally associated with gene repression [5, 6]. Oftentimes, $\mathrm{CpG}$ methylation within the gene body itself and outside of CpG islands of gene resulted in its activation [7]. DNA methylation patterns are essential for mammalian development and are faithfully maintained through cell division by DNA methyltransferases [8]. This DNA methylation can silence the gene expression and is imperative in keeping genome stability. Increasing risk for a disease onset was recorded when these epigenetic patterns are not maintained [9]. Methyltransferases and demethylases are family of proteins (enzymes) that add or remove methyl group to the cytosine of the newly synthesized DNA. Four methyltransferases were identified in human and mouse, DNAmethyltransefase3 (DNMT3) family Dnmt3a, and Dnmt3b accountable for setting up methylation of DNA and DNMT1 keeping up the DNA methylation, but DNMT2 was found to have little methyltransferase activity. by in vitro trials, all the DNA methyltransferase mRNA- sequences cloned in bovines, sheep and chicken [10]participate very strong homology with those of mouse and human, that shows a conservative role of DNA methyltransferase in various animals. Different isoforms of Dnmt1, Dnmt3a, and Dnmt3b were also found in bovine; even so the functions of these isoforms in DNA methylation stay unknown $[11,12]$.

\subsection{Histone modifications}

The network of DNA and histone proteins that can be built within the nucleus of eukaryotic cells define the chromatin. It specifies a scaffold for the wrapping of the whole genome. Nucleosome is the primary working unit of chromatin; it contains 147 base pairs of DNA, which are wrapped all over a histone octamer that exists in two copies each of histones $\mathrm{H} 2 \mathrm{~A}, \mathrm{H} 2 \mathrm{~B}$, $\mathrm{H} 3$ and H4. Unusual DNA, the histones can be covalently adapted in different manners including acetylation, methylation, sumoylation, phosphorylation, and unbiquitination [13].

\subsection{Chromatin remodeling}

The gene expression is influenced essentially by chromatin structure. The euchromatin and heterochromatin in the eukaryotic genome designate for the transcriptionally active and inactive domains, separately [14, 15]. DNA methylation [16], histone modification[17], and ATP-dependent chromatin-remodeling structure [18] are three major factors that govern the assemblage and normal of chromatin structure.

\subsection{RNA epigenetics}

Cellular RNAs carry diverse chemical modifications that used to be regarded as static and having minor roles in 'fine-tuning' structural and functional properties of RNAs [19]. The recent development of novel transcriptome-wide approaches to capture global m5C and m6A RNA methylomes has not only restored scientific interest in the field but also contributed to a better understanding how gene expression is regulated at different levels. The importance of a tightly controlled deposition of both $\mathrm{m} 5 \mathrm{C}$ and m6A into RNA is further underscored by the strong link of loss-of-function mutations in methylating and demethylating enzymes to several severe human diseases [20]. Recent studies have discovered protein 'writers', 'erasers' and 'readers' of this RNA chemical mark, as well as its dynamic deposition on mRNA and other types of nuclear RNA. These findings strongly indicate dynamic regulatory roles that are analogous to the well-known reversible epigenetic modifications of DNA and histone proteins[21].

\section{Methyl donors and methylation processing}

Methylation of $\mathrm{CpG}$ dinucleotide orders methyl donators from dietary elements suchas folate, choline and betaine, which play key roles in restoring methionine (MET) following transmethylation, the transfer of a methyl $(-\mathrm{CH} 3)$ group from the stimulated form of MET (S-adenosylmethionine; SAM) to a donee molecule. S-Adenosyl-methionine is synthesized from methionine by S-adenosylmethionine synthase (also known as methionine-adenosyl-transferase [22]. Transmethylation is needed in at least 100 various metabolic reactions [23] and is necessary for various metabolic actions including regulation of DNA expression, muscle contraction, hormonal signaling, neurotransmission, cell membrane integrity, cell growth and protein synthesis. It is notable that the multiplication of lymphocytes and macrophages is also influenced by methylation reactions. The product of transmethylation, S-adenosylhomocysteine (SAH), is changed to homocysteine (HCY), that can either be irreversibly lost from the cycle by trans-sulfuration to cysteine (CYS) or remethylated to MET by using methyl groups obtained from 5-methyl-tetrahydrofolate (5m THF), a byproduct of folate, choline or betaine. Under normal situation, diminished store of MET to the cycle will decline SAM concentration which will favor remethylation by reducing SAMmediated inhibition of Methylene tetrahydrofolate reductase (MTHFR) and Betaine-homocysteine methyltransferase (BHMT), and decrease trans-sulfuration by withdrawingCystathionine beta synthase (CBS) activation. A lack of methyl donors will have the same impact on these enzymes, and HCY will aggregate because of a lack of sufficient methyl groups for remethylation and low CBS activity [24].

Some essential roles of some methyl donors in animal body.

The first methyl donor for DNA methylation is S-adenosylmethionine (SAM), a type generated in the cyclical cellular procedure called one-carbon metabolism (transfer). One-carbon metabolism is catalyzed by several enzymes in the presence of dietary micro nutrients, including folate, choline, betaine and other B vitamins. For this reason, nutrition status, particularly micronutrient intake, has been a focal spot when studying epigenetic mechanisms. [25].

\subsection{Methionine (Met)}

From the nutritional point of view, Met is classified as essential (carbon skeleton cannot be synthesized by the body) sulfur-containing AA that tasks many roles in the body including(1) participation in protein synthesis and the 
production of other sulfur-containing amino acids (e.g. homocysteine - a sulfur-containing AA which is an indirect product of methylation and trans-sulfuration, in what is called recently "protein related role of AA" [26, 27], (2) acting as a precursor of carnitine and glutathione, thus protect cells against oxidative stress [28, 29], 3) labile methyl group (CH3) is incriminated in the metabolism of energy, which have to be provided from the diet [30]. Under the influence of methionine-adenosyl transferase, Met is converted to S-adenosyl-methionine (SAM). S-adenosyl-methionine is a common co-substrate that supplies methyl groups required for various metabolic processes, including DNA methylation and synthesis of RNA, proteins and lipids. In the process of methylation, SAM is converted to S-adenosyl-homocysteine in the presence of methyltransferases "non-protein related role of AA" [22, 31]. Cysteine (Cys) is grouped d as semi essential because it can be produced from Met [32]. The concept of alternate dietary methyl donors (i.e. betaine, choline, methylneogenesis precursors) sparing the methionine requirement has recently been discussed [33]. The inverse association between plasma betaine and homocysteine levels was most noticeable when serum folate levels were low [34]. Because -in human- betaine [35], choline [36] and folic acid [37], be able effectively y lessen plasma homocysteine by enlarging remethylation to methionine, these nutrients are very likely to be able sparing the methionine demand. Actually, in smart isotope studies the choline and folic acid doubled remethylation to methionine in methionine deficient diets. That idea can be applied by using fewer costly additives containing choline and/or betaine in poultry diets. Several studies proved that betaine and/or choline can reserve methionine demand in growing chickens [38] and young pigs [39], not only for the synthesis of protein but also for creatine and carnitine synthesis. The fact that these studies were able to show a sparing effect of methyl groups on the methionine requirement demonstrates the enormous demands of these pathways.

\subsection{Choline and betaine (N,N,N-trimethy-lglycine)}

Choline and betaine are dietary leading nutrients with several biological functions. Along with folate and other B vitamins, choline and betaine contribute to one-carbon metabolism that constitutes a network of integrated biochemical pathways transferring methyl groups from one compound to another [40,41]. Choline is oxidized to betaine, both folate and betaine donate methyl groups to homocysteine and produce methionine, which transfer a methyl-group to S-adenosyl-methionine (a universal methyl-group donor) for methylation of DNA and RNA. Through this process, one-carbon metabolism affects genomic stability and expression and intervenes nucleotide synthesis.Thus, a number of animals in vivo and, to a lesser extent, human studies have investigated the role of dietary choline and/or betaine and their impact on global and candidate gene DNA methylation. As, choline-deficient diet lessens hepatic folate levels in animals [42]. Parallel, a folate deficient diet lowered hepatic choline levels in animals [43]. A double-blind clinical study of folate supplementation raised plasma betaine levels in a dose-dependent style following folate supplement [44]. Plasma choline levels existed reactive to reduction and subsequent repletion of folate intake [45]. Betaine and choline have additional biological galas not shared by each other. Choline is a precursor for phosphatidylcholine and sphingomyelin, which are integrated into cellular membrane and involved in signal transduction [46]. Choline is also a pioneer for the neurotransmitter acetylcholine and is concerned in brain development and normal memory job [47, 48].

Betaine is an osmolyte (play a role in maintaining cell volume, fluid balance and protects cells, proteins, and enzymes from water stress[49,50]. Betaine supplementation plays crucial roles in nutritional programming through epigenetic regulation of gene expression in pigs, ducks meat and egg type chickens. Betaine supplement was associated with retained positional effects in the -rate limiting enzyme- lipoprotein lipase (LPL) gene promoter, as measured in adipocytes [51]. With addition of betaine, most of the promoters were highly hypo-methylated and many $\mathrm{CpG}$ sites were built to be hyper methylated. Interestingly, LPL gene expression was significantly decreased with betaine supplementation. Maternal betaine supplementation enhances betaine/methionine metabolism and DNA methyltransferase expression, causes hypermethylation of the differentially methylated region (DMR) on IGF2 gene, which was associated with augmented expression of insulin like growth factor II (IGF2) and cell proliferation/anti-apoptotic markers in the hippocampus of neonatal piglets [52]. Dietary betaine supplementation $(3 \mathrm{~g} / \mathrm{kg}$ diet) during gestation of sows, attenuates hepatic lipogenesis in neonatal piglets via epigenetic and GR-mediated mechanisms [53]. Since one-carbon (1C) metabolism consists of an integrated series of metabolic pathways that include the folate cycle and methionine remethylation and trans-sulfuration pathways, maternal nutrient status can cause epigenetic alterations to the genome of the developing fetus, which potentially can impact future generations [54].

\subsection{Folic acid (Folate)}

folate (B9), riboflavin (B2) cobalamin (B12), serve as cofactors in (1C) metabolismwhile pyridoxine (B6) is essential for transsulfuration pathway, folate work as a catalytic substrate for the transfer of (1C) units [25, 32]. Numerous reports have indicated a link among folate, choline, and lipid metabolism. Folate has a role in the maintenance of cellular S- Adenosylmethionine (AdoMet) and Adenosyl-homocysteine (AdoHcy) levels. Dietary folate play a role in epigenetic mechanisms, in the liver, that responsible for many methylation reactions which are used for post translational modification of proteins, methylation of DNA, and the synthesis of hormones, creatine, carnitine, and phosphatidylcholine PC. The tetra-hydro-folate (THF) plays a crucial role in a number of reactions that generate methyl groups from the catabolism of sarcosine, serine, dimethylglycine, and glycine [6]. Recently, folate status may be influencing microRNA expression linked to the severity of fatty liver disease. MicroRNAs (miRNAs) are short, non-coding transcripts, of closely 22 nucleotides in length. They belong to a regulative class of RNAs which repress expression of target mRNA. Folic acid supply controls the expression of miRNAs perhaps by changes in methylation levels of promoter regions in the genome [55]. The severity of Non-alcoholic fatty liver disease (NAFLD) induced by a choline- and folate-deficient diet in mice is associated with altered expression of hepatic miRNAs, including miR-181a, miR-34a, miR-200b, and miR-221 [56]. Moreover, maintenance and regulation of the epigenetic state which depends on (1C) metabolism require adequate provision of B vitamins (including folate, vitamin B6 and vitamin B12), glycine, methionine, serine, choline, histidine, and creatine [57]. These nutrients play an important role in regulating the availability of S-adenosyl-methionine (a major methyl donor for DNA and protein methylation by specific 
DNA and protein methyltransferases [58]. For example, essential B vitamins, folate, and methionine deficient diet during the breeding period in sheep resulted in altered DNA methylation, insulin resistance, and higher blood pressure mostly observed in adult male kids [59].

\subsection{Vitamins $\mathrm{B} 2, \mathrm{~B} 6$ and $\mathrm{B} 12$}

The water-soluble vitamins B2, B6 and B12 have an essential catalytic role in folate and one-carbon metabolism. Vitamin B6 serves as a coenzyme to serine hydroxyl-methyltransferase, the vital enzyme in the folate cycle converting THF to 5,10methylene THF [60]. Riboflavin, or vitamin B2, is a precursor for Flavin-adenine-dinucleotides, which is a cofactor to MTHFR, the enzyme responsible for the reduction of 5,10-methylene THF to 5-methyl THF[61].Vitamin B12 is the coenzyme of methionine synthase, which catalyzes the reaction of homocysteine, the by-product of SAM, to methionine. Therefore consumption of these water-soluble B vitamins has the potential to affect the efficiency of the one-carbon metabolism pathway. Both animal and human studies have evaluated the role of vitamin B12 in DNA methylation profiles. An in vivo rat model showed that deprivation of vitamin B12 in addition to a diet supplemented with folate enhanced placental global hypo-methylation compared to a diet exclusively supplemented with folate, suggesting that the interaction between micronutrients can alter methylation patterns more profoundly than excess or deprivation of just one micronutrient [62]. vitaminB12 and folatesupplemented rat maternal diet, increases methylation of DNA of the agouti gene in the offspring, preventing the development of obesity [63]. The effect of maternal diet supplementation with methyl donors can be succeed to the next era through germline epigenetic modifications [64]. Consequently, the nutritional and physiological state of the animal will alter the production of these bioactive substances, thus regulating the availability of methyl donors. When cysteine or taurine is deficient in the diet, their synthesis from methionine will be increased in vivo, thus decreasing total Sadenosylmethionine availability for DNA or protein methylation. Inadequate synthesis of glycine and serine, coupled with low supplies from the diet, can also impair (1C) metabolism [65]. So, an essential AA deficiency can alter the epigenetic state by changes in DNA methylation as well as histone modifications [66].

\section{Closing Remarks}

Biochemical interactionscan direct Cross-talk between nutrients (micronutrients) and gene expression, Because methylation is a cell type dependent, a comprehensive epigenetic analysis remains an optimal approach. Future studies should continue to focus on tissue specificity in DNA methylation investigation. To apply the rational and economic use of the most expensive micro-nutrients in the animals and poultry diets, adoption of studying nutri-epigenetics in production trials might help understanding productive phenotypes and re-estimation of dietary requirements of essential nutrients. One-carbon metabolism role perform an outstanding role in DNA synthesis and in validating and maintaining the epigenetic sketch via their diverse metabolic intermediates and contributing 1C units. Thus, an impaired 1C metabolic pathway may cause changes to intracellular methyl pools that limit the availability of methyl groups for relevant DNA and histone modifications. There are two interacting factors that can affect this methyl puddle 1) the dietary provision of methyl donors (folate) and 1C metabolic cofactors (vitaminB12), 2) nature and extent of polymorphic variations in genes encoding $1 \mathrm{C}$ metabolic enzymes. Abnormal $1 \mathrm{C}$ metabolism can lead to aberrant methylation patterns in DNA and associated histone proteins that affect genome stability, gene expression, cellular differentiation and long-term development [67]. Either under nutrition or over nutrition during pregnancy (peri-conceptional time), remains a important problem in both medicine and animal agriculture as it can results in epigenetic modifications of some genes in both animals and humans. These changes are influenced by various factors (severity of malnutrition, sex, and gestational period) and may persist in offspring during postnatal lifetime and may transport to the next generation. Nutrients, particularly amino acids and B vitamins, are essential for the regulation of epigenetics. Binding evidence indicates that, the fetal and early neonatal ages of development are radically sensitive to environmental implies that have long-lasting consequences' to postnatal growth, health, and likely athletic performance.

Conflict of interest statement

The authors declare that there are no conflicts of interest

\section{References}

[1]. Deans, C. and K.A. Maggert, What do you mean "epigenetic"? Genetics, 2015. 199(4): p. 887-896.

[2]. Trošelj, K.G., R.N. Kujundžić, and I. Grbeša, Epigenetics and Gene Physiology. 2009.

[3]. Preedy, V.R., R.R. Watson, and C.R. Martin, Handbook of behavior, food and nutrition. 2011: Springer Science \& Business Media.

[4]. Choi, S.-W. and S. Friso, Nutrients and epigenetics. 2009: CRC Press.

[5]. François, M., et al., G-quadruplexes: A possible epigenetic target for nutrition. Mutation Research/Reviews in Mutation Research, 2015. 764: p. 101-107.

[6]. Crider, K.S., et al., Folate and DNA methylation: a review of molecular mechanisms and the evidence for folate's role. Advances in Nutrition: An International Review Journal, 2012. 3(1): p. 21-38.

Jones, P.A., Functions of DNA methylation: islands, start sites, gene bodies and beyond. Nature Reviews Genetics, 2012. 13(7): p. 484-492.

Schübeler, D., Function and information content of DNA methylation. Nature, 2015. 517(7534): p. 321-326.

Zykovich, A., et al., Genome-wide DNA methylation changes with age in disease-free human skeletal muscle. Aging cell, 2014. 13(2): p. 360-366.

[10]. $\quad$ Khatib, H., Livestock epigenetics. 2012: John Wiley \& Sons.

[11]. Golding, M.C. and M.E. Westhusin, Analysis of DNA (cytosine 5) methyltransferase mRNA sequence and expression in bovine preimplantation embryos, fetal and adult tissues. Gene Expression Patterns, 2003. 3(5): p. 551-558.

[12]. Russell, D.F. and D. Betts, Alternative splicing and expression analysis of bovine DNA methyltransferase 1. Developmental Dynamics, 2008. 237(4): p. 1051-1059.

[13]. Tessarz, P. and T. Kouzarides, Histone core modifications regulating nucleosome structure and dynamics. Nature reviews Molecular cell biology, 2014. 15(11): p. 703-708

[14]. Bernardi, G. Genome Organization and Chromosome Architecture. in Cold Spring Harbor symposia on quantitative biology. 2016. Cold Spring Harbor Laboratory Press.

[15]. Sadoni, N., et al., Nuclear organization of mammalian genomes Polar chromosome territories build up functionally distinct higher order compartments. The Journal of cell biology, 1999. 146(6): p. 1211-1226.

16]. Suzuki, M.M. and A. Bird, DNA methylation landscapes: provocative insights from epigenomics. Nature Reviews Genetics, 2008. 9(6): p. 465-476.

[17]. Wang, Y., et al. Linking covalent histone modifications to epigenetics: the rigidity and plasticity of the marks. in Cold Spring Harbor symposia on quantitative biology. 2004. Cold Spring Harbor Laboratory Press

Schwartzentruber, J., et al., Driver mutations in histone H3. 3 and chromatin remodelling genes in paediatric glioblastoma. Nature, 2012. 482(7384): p. 226-231.

[19]. Liebers, R., M. Rassoulzadegan, and F. Lyko, Epigenetic regulation by heritable RNA. PLoS Genet, 2014. 10(4): p. e1004296. 
[20]. Blanco, S. and M. Frye, Role of RNA methyltransferases in tissue renewal and pathology. Current opinion in cell biology, 2014. 31: p. 1-7.

[21]. Fu, Y., et al., Gene expression regulation mediated through reversible m6A RNA methylation. Nature Reviews Genetics, 2014. 15(5): p. 293-306.

[22]. Wu, G., Functional amino acids in nutrition and health. Amino Acids, 2013. 45(3): p. 407-411.

[23]. Sharma, P., et al., Mining literature for a comprehensive pathway analysis: a case study for retrieval of homocysteine related genes for genetic and epigenetic studies. Lipids in health and disease, 2006. 5(1): p. 1.

[24]. Cronje, P., Essential role of methyl donors in animal productivity. Animal Production Science, 2016

[25]. Anderson, O.S., K.E. Sant, and D.C. Dolinoy, Nutrition and epigenetics: an interplay of dietary methyl donors, one-carbon metabolism and DNA methylation. The Journal of nutritional biochemistry, 2012. 23(8): p. 853-859.

[26]. Bertolo, R.F. and L.E. McBreairty, The nutritional burden of methylation reactions. Current Opinion in Clinical Nutrition \& Metabolic Care, 2013. 16(1): p. 102-108.
Bertolo, R.F. and L.E. McBreairy, The mutritional burcen of methylation reactions. Current Opinion in Clinical Nutrition \& Metabolic Care, 2013. Troen, A.M., et al., The atherogenic effect of excess methionine intake. Proceedings of the National Academy of Sciences, 2003. 100(25): p. 1508915094.

[28]. Wu, G., Amino acids: metabolism, functions, and nutrition. Amino acids, 2009. 37(1): p. 1-17.

[29]. Li, P., et al., Amino acids and immune function. British Journal of Nutrition, 2007. 98(02): p. 237-252.

[30]. Ratriyanto, A. and R. Indreswari, Effects of Protein Levels and Supplementation of Methyl Group Donor on Nutrient Digestibility and Performance of Broiler Chickens in the Tropics. International Journal of Poultry Science, 2014. 13(10): p. 575.

[31]. WU, B.-y., et al., Effect of methionine deficiency on the thymus and the subsets and proliferation of peripheral blood T-cell, and serum IL-2 contents in broilers. Journal of Integrative Agriculture, 2012. 11(6): p. 1009-1019.

[32]. Baker, D.H., Advances in protein-amino acid nutrition of poultry. Amino acids, 2009. 37(1): p. 29-41

[33]. Fukagawa, N.K., Sparing of methionine requirements: evaluation of human data takes sulfur amino acids beyond protein. The Journal of nutrition, 2006. 136(6): p. 1676S-1681S.

[34]. Fernàndez-Roig, S., et al., Low folate status enhances pregnancy changes in plasma betaine and dimethylglycine concentrations and the association between betaine and homocysteine. The American journal of clinical nutrition, 2013. 97(6): p. 1252-1259.

[35]. Ueland, P.M., P.I. Holm, and S. Hustad, Betaine: a key modulator of one-carbon metabolism and homocysteine status. Clinical Chemical Laboratory Medicine, 2005. 43(10): p. 1069-1075.

[36]. Olthof, M.R., et al., Choline supplemented as phosphatidylcholine decreases fasting and postmethionine-loading plasma homocysteine concentrations in healthy men. The American journal of clinical nutrition, 2005. 82(1): p. 111-117.

[37]. Zhang, C., et al., Association between B vitamins supplementation and risk of cardiovascular outcomes: a cumulative meta-analysis of randomized controlled trials. PloS one, 2014. 9(9): p. e107060.

[38]. Zhan, X., et al., Effects of methionine and betaine supplementation on growth performance, carcase composition and metabolism of lipids in male broilers. British poultry science, 2006. 47(5): p. 576-580.

[39]. Fernandez-Figares, I., et al., Effect of dietary betaine on nutrient utilization and partitioning in the young growing feed-restricted pig. Journal of animal science, 2002. 80(2): p. 421-428.

[40]. Mason, J.B., Biomarkers of nutrient exposure and status in one-carbon (methyl) metabolism. The Journal of nutrition, 2003. 133(3): p. 941S-947S.

[41]. Mostowska, A., et al., Folate and choline metabolism gene variants and development of uterine cervical carcinoma. Clinical biochemistry, 2011. 44(8): p. 596-600.

[42]. Zeisel, S.H., Dietary choline deficiency causes DNA strand breaks and alters epigenetic marks on DNA and histones. Mutation Research/Fundamental and Molecular Mechanisms of Mutagenesis, 2012. 733(1): p. 34-38.

[43]. Silva, R.P., et al., Novel insights on interactions between folate and lipid metabolism. Biofactors, 2014. 40(3): p. 277-283.

[44]. Hall, M.N., et al., Supplementation with Folic Acid, but Not Creatine, Increases Plasma Betaine, Decreases Plasma Dimethylglycine, and Prevents a Decrease in Plasma Choline in Arsenic-Exposed Bangladeshi Adults. The Journal of nutrition, 2016. 146(5): p. 1062-1067.

[45]. $\quad$ Cordero, P., et al., Dietary supplementation with methyl donors reduces fatty liver and modifies the fatty acid synthase DNA methylation profile in rats fed an obesogenic diet. Genes \& nutrition, 2013. 8(1): p. 105-113.

[46]. Zeisel, S.H., Dietary Choline, Betaine, Methionine, and Epigenetic Mechanisms Influencing Brain Development. Nutrition in Epigenetics, 2011: p. 225-240.

[47]. Meck, W.H. and C.L. Williams, Metabolic imprinting of choline by its availability during gestation: implications for memory and attentional processing across the lifespan. Neuroscience \& Biobehavioral Reviews, 2003. 27(4): p. 385-399.

[48]. Jiang, X., A.A. West, and M.A. Caudill, Maternal choline supplementation: a nutritional approach for improving offspring health? Trends in Endocrinology \& Metabolism, 2014. 25(5): p. 263-273.

[49]. Burg, M.B. and J.D. Ferraris, Intracellular organic osmolytes: function and regulation. Journal of Biological Chemistry, 2008. 283(12): p. 73097313 .

[50]. Lukić, M., et al., The effect of full substitution of supplemental methionine with betaine in broiler nutrition on production and slaughter results. Biotechnology in Animal Husbandry, 2012. 28(2): p. 361-368.

[51]. Xing, J., L. Kang, and Y. Jiang, Effect of dietary betaine supplementation on lipogenesis gene expression and CpG methylation of lipoprotein lipase gene in broilers. Molecular biology reports, 2011. 38(3): p. 1975-1981.

[52]. $\quad \mathrm{Li}, \mathrm{X}$., et al., Dietary betaine supplementation to gestational sows enhances hippocampal IGF2 expression in newborn piglets with modified DNA methylation of the differentially methylated regions. European journal of nutrition, 2015. 54(7): p. 1201-1210.

[53]. Cai, D., et al., Gestational dietary betaine supplementation suppresses hepatic expression of lipogenic genes in neonatal piglets through epigenetic and glucocorticoid receptor-dependent mechanisms. Biochimica et Biophysica Acta (BBA)-Molecular and Cell Biology of Lipids, 2016. 1861(1): p. $41-50$.

[54]. Funston, R. and A. Summers, Epigenetics: setting up lifetime production of beef cows by managing nutrition. Annu. Rev. Anim. Biosci., 2013. 1(1): p. 339-363.

[55]. Fuentes, E., et al., Mechanisms of chronic state of inflammation as mediators that link obese adipose tissue and metabolic syndrome. Mediators of inflammation, 2013. 2013.

[56]. Tryndyak, V.P., et al., Plasma microRNAs are sensitive indicators of inter-strain differences in the severity of liver injury induced in mice by a choline-and folate-deficient diet. Toxicology and applied pharmacology, 2012. 262(1): p. 52-59.

[57]. Wang, J., et al., Nutrition, epigenetics, and metabolic syndrome. Antioxidants \& redox signaling, 2012. 17(2): p. 282-301.

[58]. Lin, G., et al., Improving amino acid nutrition to prevent intrauterine growth restriction in mammals. Amino Acids, $2014.46(7)$ : p. 1605-1623.

[59]. Dominguez-Salas, P., et al., Maternal nutritional status, C 1 metabolism and offspring DNA methylation: a review of current evidence in human subjects. Proceedings of the Nutrition Society, 2012. 71(01): p. 154-165.

[60]. Perry, C., et al., Effect of vitamin B 6 availability on serine hydroxymethyltransferase in MCF-7 cells. Archives of biochemistry and biophysics, 2007. 462(1): p. 21-27.

[61]. Chan, J., et al., Low dietary choline and low dietary riboflavin during pregnancy influence reproductive outcomes and heart development in mice. The American journal of clinical nutrition, 2010. 91(4): p. 1035-1043.

[62]. Kulkarni, A., et al., Effects of altered maternal folic acid, vitamin B 12 and docosahexaenoic acid on placental global DNA methylation patterns in Wistar rats. PLoS One, 2011. 6(3): p. e17706.

[63]. Jirtle, R.L. and F.L. Tyson, Environmental epigenomics in health and disease. 2013: Springer.

[64]. Cui, H., et al., Loss of IGF2 imprinting: a potential marker of colorectal cancer risk. Science, 2003. 299(5613): p. 1753-1755.

[65]. $\quad \mathrm{Wu}, \mathrm{G}$. , et al., Board-invited review: intrauterine growth retardation: implications for the animal sciences. Journal of animal science, 2006. 84(9): p. 2316-2337.

[66]. Oommen, A.M., et al., Roles for nutrients in epigenetic events. The Journal of nutritional biochemistry, 2005. 16(2): p. $74-77$.

[67]. Xu, J. and K.D. Sinclair, One-carbon metabolism and epigenetic regulation of embryo development. Reproduction, Fertility and Development, 2015. 27(4): p. 667-676. 